\title{
Identification of Novel Membrane Structures in \\ Plasmodium falciparum Infected Erythrocytes
}

\section{Carlos A Clavijo, Carlos A Mora, Enrique Winograd ${ }^{* /+}$}

Laboratorio de Biología Celular, Instituto Nacional de Salud, Avenida El Dorado con Carrera 50, Bogotá, Colombia *Departamento de Química, Universidad Nacional de Colombia, Bogotá, Colombia

Little is known about the molecular mechanisms underlying the release of merozoites from malaria infected erythrocytes. In this study membranous structures present in the culture medium at the time of merozoite release have been characterized. Biochemical and ultrastructural evidence indicate that membranous structures consist of the infected erythrocyte membrane, the parasitophorous vacuolar membrane and a residual body containing electron dense material. These are subcellular compartments expected in a structure that arises as a consequence of merozoite release from the infected cell. Ultrasurface membrane. Since these me brane modificetions are detected only a fer merozoites have been releas f from the infected erythrocyte, it is proposed that they might play a role in the relesse of mero released from the infected
zoites from the host cell.

Key words: Plasmodium falciparum - parasite release - membrane fusion - parasitophorous vacuolar membrane

The erythrocytic life cycle of the human malarial parasite Plasmodium falciparum is responsible for most of the pathology and mortality associated with this disease (Miller et al. 1994). The cycle is initiated by entry of a merozoite into the host red blood cell by invagination of the erythrocyte plasma membrane (Ward et al. 1993, Dluzewski et al. 1995). During the next $48 \mathrm{hr}$, the intracellular parasite develops surrounded by two membranes: the erythrocyte plasma membrane and the invaginated membrane closely apposed to the parasite itself, the parasitophorous vacuolar membrane. During the first $20 \mathrm{hr}$ of development, the young feeding parasite (trophozoite) is observed as a "ring form". After this time, an increase in various metabolic activities including the degradation of hemoglobin in digestive vacuoles takes place. Digestion of hemoglobin results in the production of amino acids and an insoluble pigment called hemozoin, which accumulates in and is characteristic of the mature trophozoite stage. During

This investigation received financial support from the UNDP/World Bank/WHO Special Programme for Research and Training in Tropical Diseases (TDR), the Colombian Institute for the Development of Science and Technology "Francisco Jose de Caldas" COLCIENCIAS

Corresponding author. Fax: +571-222.3055

Accepted 20 August 1997 the last $14 \mathrm{hr}$ of development, DNA replication and cellular differentiation of 16 to 32 daughter cells or merozoites occurs by asexual division or schizogony. Hemozoin concentrates within a residual body, before merozoites are released from the red cell into the bloodstream.

Little is known about the molecular mechanisms underlying the release of merozoites from malaria infected erythrocytes. Video-microscopy studies have shown that the actual rupture of a schizont-infected erythrocyte is preceded by swelling and vesiculation of the host cell membrane, and then merozoites are released with explosive suddenness (Dvorak et al. 1975, Hermentin \& Enders 1984). Malarial proteases have been implicated in merozoite release, since rupture of infected erythrocytes can be prevented through the use of protease inhibitors (Banyal et al. 1981, Hadley et al. 1983, Lyon \& Haynes 1986). Furthermore, plasmodial proteases with activities against known red cell membrane skeletal proteins, have been isolated and characterized (Deguercy et al. 1990); however it has not been determined whether these proteases actually participate in the rupture of the red cell membrane during merozoite escape. At the time when schizogony is nearly complete, the energetic charge of the infected erythrocyte falls (Yamada \& Sherman 1980). Such a reduction in ATP levels could disturb the osmotic duction in ATP levels could disturb the osmotic
gradient across the erythrocytic membrane that is gradient across the erythrocytic membrane that is bly, pore forming proteins which have been re- 
ported in other parasitic protozoa (Noronha et al. 1994, Andrews 1994), could function in the escape of merozites from the infected cell, but these are yet to be identified in the case of malarial para-

In this study, a preliminary characterization of membranous structures found in the culture medium at the time when merozites are released from infected erythrocytes has been carried out. It is shown that membranous structures are composed of a surface membrane, a parasitophorous vacuolar membrane, a residual body containing electron-dense material, and a novel structure which extends from the former parasite compartment to the surface membrane. Our results suggest a possible function of these novel membrane modifications in the process of parasite escape from the host cell.

\section{MATERIALS AND METHODS}

Isolation of membranous structures - $P$. falciparum cultures (Trager \& Jensen 1976) were synchronized by gelatin flotation (Jensen 1978) followed by sorbitol lysis (Lambros \& Vandenberg 1979) of mature infected erythrocytes. Parasite maturation was monitored every $8 \mathrm{hr}$ by Giems staining of thin blood-smears. When parasite reached the ring stage, the culture was centrifuge at $230 \mathrm{xg}$ for $5 \mathrm{~min}$. The supernatant containing for 10 min, and the in a small volu $10 \mathrm{mM}$ sodiususpe buffer $\mathrm{pH} 7.4$ co of 10 mM sodium phosphase The suspension of membran The suspension of membranous structures wa posed of 5 and 13\% Percoll cushions (vol/vol in PBS), and centrifugation at $900 \times$ g for 15 min wa carried out. Membranous structures were isolted from the 5-13\% Percoll interphase and three times in PBS.

Fluorescence microscopy - Freshly isolated membranous structures were processed for immunofluorescence microscopy as previously describe (Winograd \& Sherman 1989). Rabbit anti-erythroid spectrin was kindly provided by $\mathrm{Dr} \mathrm{M}$ Wasserman, and monoclonal antibody 8E7/55 against the parasitophorous vacuolar antigen QF116 was a kind gift from Dr AR Hibbs. Staining with merocyanine 540 was essentially carrie out as previously reported (Kara et al. 1988). Membranous structures were labeled with quinacrine by incubation in a $0.1 \%(\mathrm{w} / \mathrm{v})$ quinacrine solution in Hepes Buffered Saline (HBS; $10 \mathrm{mM}$ Hepes buffer $\mathrm{pH}=7.40$ containing $0,15 \mathrm{M} \mathrm{NaCl}$ ) for $10 \mathrm{~min}$ a $37^{\circ} \mathrm{C}$. After washing in HBS, cells were examine by fluorescence microscopy. As a control, live infected erythrocytes were labeled with quinacrin at different stages of development. In every instance, infected erythrocytes presented a strong beled withe signal. Erythrocytes were surface lawith $P$. falciparum as described above. Membra with $P$. falciparum as described above. Membrareached the ring stage of development. Membranebound biotin was detected by fluorescence microscopy after incubation in streptavidin-fluorescein. Electron microscopy - Membranous structure Electron microscopy - Membranous structures
were purified by discontinuous Percoll gradients as described above, and immediately fixed for $1 \mathrm{hr}$ in $2 \%$ glutaraldehyde in PBS at room temperature. Membranous structures were then processed either for transmission (Winograd \& Sherman 1989) or scanning electron microscopy (SEM) (Gruenber et al. 1983) and observations were either carried out on a Phillips transmission electron microscope Model CM10 or a Phillips scanning electron microscope Model 515 .

\section{RESULTS}

To learn more about the process of merozoit release from malaria infected erythrocytes, we carried out a characterization of the membranous stripar in the culture muber of membranous structures shown), coincident with the time of merozote lease (i.e the tempos interphase betwen the schizo (ich ring stages), sugesting that the sthtures are implicated in the process of merozote release from the infected erythrocyte.

Observations by phase contrast microscopy, showed that membranous structures have morphology and dimensions similar to that of an infected erythrocyte, including the presence of a residua . ermost membrane reacted with antibodies directed against erythroid spectrin (Fig. 1B). Furthermore, when the erythrocyte membrane was labeled with biotin prior to infection, streptavidin conjugated fluorescein bound only to the outermost membrane of the structures (results not shown). These results suggest that the external membrane of the membranous structures originates from the infected erythrocyte.

Membranous structures could be labeled with merocyanine 540 (Fig. 1D), a reagent previously shown to interact with the parasitophorous vacuolar membrane (Elford et al. 1985). In addition, membranous structures reacted with the monoclonal antibody 8E7/55 (Fig. 1F), whose specificity has been demonstrated to be against the parasitophorous vacuolar antigen $\mathrm{QFll}$ (Kara et
al. 1988). These results indicate that the
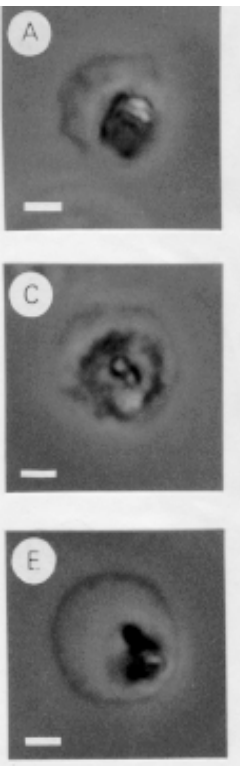
Fig.1: immunofluorescence microscopy of membranous struc-
tures. (A), (C) and (E) are phase-contrast microscopy images;
(B), (D) and (F) are the corresponding fluorescence microscopy (B), (D) and (F) are the corresponding fluorescence microscopy
photographs. In (B) the reaction with anti-erythroid spectrin is hown. In (D) merocyanine 540 staining and in (F) monoclonal antibody $8 \mathrm{E} 7 / 55$ are used to stain the parasitophorous vacu-
olar membrane. Note that the staining pattern generated either with merocyanine 540 or monoclonal antibody $8 \mathrm{E} 7 / 55$ is different from that with anti-erythroid antibodies. The former repretion of the parasitophorous vacuolar membrane, while the later appears to correspond with the surface membrane. Bars $=2 \mu \mathrm{m}$.

parasitophorous vacuolar membrane is also an mportant component of membranous When membranous structures were incubated with quinacrine no fluorescent signal was detected, suggesting that membranous structures are devoid of DNA.

Based on the evidence presented above, membranous structures contain subcellular compartments expected to be present in a structure that arises as a consequence of merozoite release from the infected cell, i.e. it is composed of compartments not directly involved in the differentiation of merozoites (i.e. red cell membrane, parasitophorous vacuolar membrane and residual body containing dense material). croscopy, showed that membranous structures are composed of a surface membrane with associated knobs or excrescences and a residual body containing electron-dense material (Fig. 2A). Vesicles or tubules, similar to membrane-bound structures described in schizont-infected erythrocytes (Langreth et al. 1978, Atkinson \& Aikawa 1990), were found within the space defined by the outermost membrane and the residual body (Fig. 2A). In addition, larger membrane-bound structures $(0.7$ to $1.4 \mu \mathrm{m}$ ) appearing to derive from the surface membrane, contain electron-dense material similar to the one present in the residual body (Fig. 2A). In other microscopic sections, the red cell membrane and the membrane surrounding the residual body appear to be continuous (Fig. 2B), and occasionally a well defined structure extending from the erythrocyte membrane to the residual
body was observed (Fig. 2C). These results indibody was observed (Fig. 2C). These results indicate that the former parasite compartment and the through a novel duct-like structure.

Scanning electron mi strectur. the membranous structures, showed the presence of an opening which caves in appearing to lead to a duct-like structure (Fig. 2D, E, F).

\section{DISCUSSION}

In this study, membranous structures were idenified in the culture medium of P. falciparum inected erythrocytes. The time of appearance of hese membranous structures was found to be corlated to the event when merozoites are released rom infected erythrocytes. Membranous structures were found to be composed primarily of the erythmeyte membrane, the parasitophorous vacuolar
membrane, and a residual body containing dense material. Since these subcellular compartments do not participate in the cellular differentiation of merozoites, membranous structures could correspond to cellular structures arising periodically in the culture medium as a consequence of merozoite release from infected erythrocytes.

Ultrastructural studies demonstrated the presence of a membrane extending from the former parasite compartment to the surface membrane, forming an apparent duct-like structure. The fact that these structures are not detectable on schizontinfected erythrocytes either by transmission (Langreth et al. 1978, Atkinson \& Aikawa 1990) or SEM (Gruenberg et al. 1983), suggests that the formation of such membrane modifications might be functionally related to the release of merozoites from infected erythrocytes.

In a previous study a parasitophorous duct on erythrocytes infected with mature stages of the 

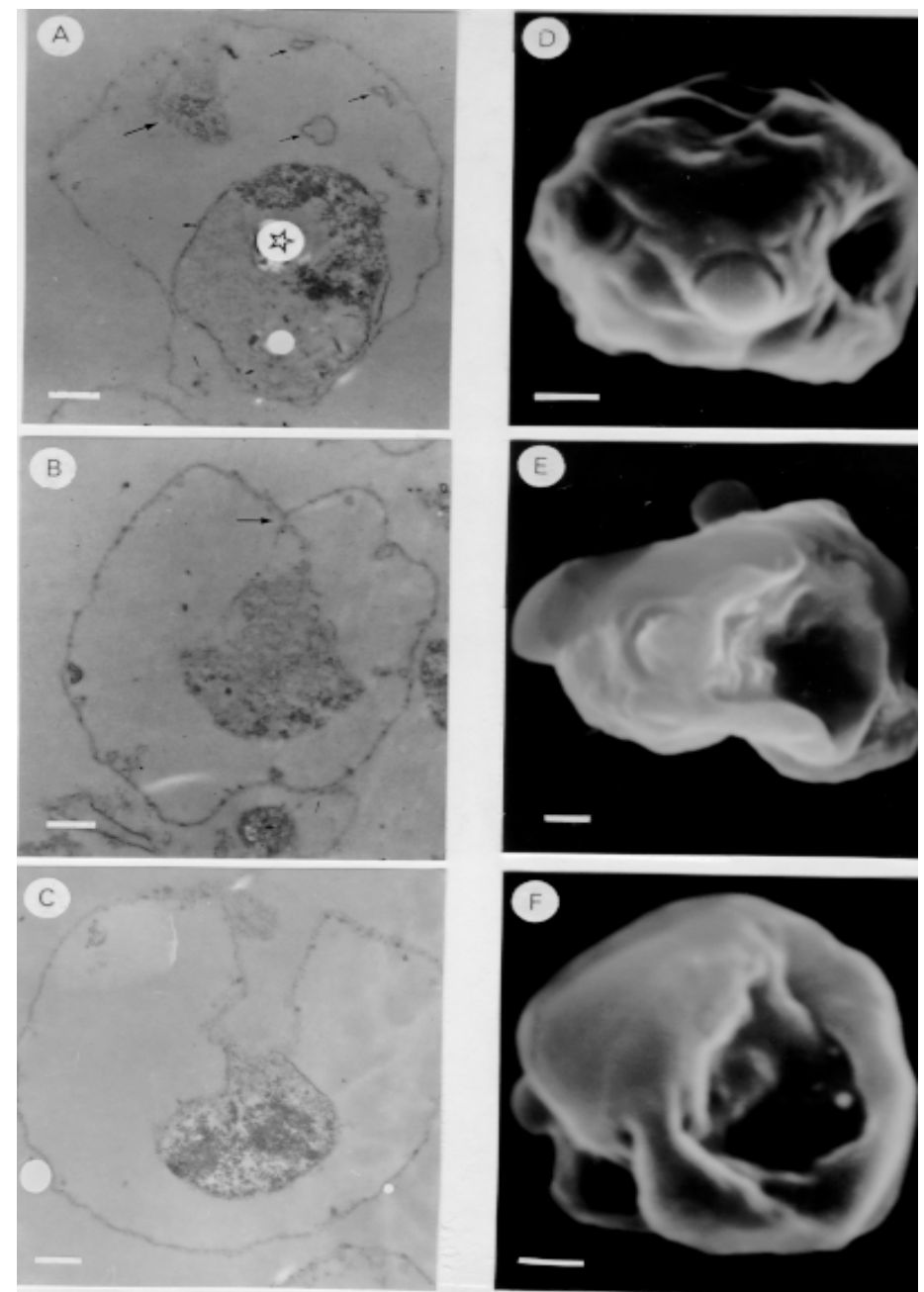

Fig. 2: electron microscopy of membranous structures. In (A), (B) and (C) transmission electron microscope images are shown;
(D) (E) and (F) were obtained using an scanning electron microscope. In (A) small vesicles or tubules are shown by short arrows and a larger membrane-bound structure (longer arrow), containing electron dense material similar to the one in the residual body (star) is also observed. In another section, the red cell membrane and the membrane surrounding the internal body converge in
common point (arrow) (B). In (C) the surface membrane and the membrane surrounding the residual body are continuos. Differen parasite, through which macromolecules present in the external culture medium could reach the intraerythrocytic paraste, was proposed (Pou the intraerythrocytic parasite, was proposed (Pouvelle evidence for a functional duct was not found.

Although the exact role of the parasitophorous duct is not currently understood, we would like to suggest that the duct identified in this study, might play a role in the release of merozoites from infected erythrocytes (Fig. 3). The model of parasite release assumes that the parasite compartment within the infected cell becomes topologically continuous with the extracellular environment as a result of a membrane fusion even

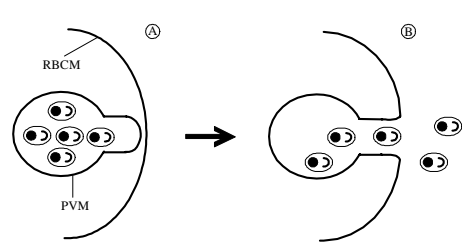

Fig. 3: a model of parasite release in malaria infected erythrocytes. A schizont infected erythrocyte is shown in (A). Upon membrane fusion between the parasitophorous vacuolar membrane (PVM) and the red blood cell membrane (RBCM), meroas depicted in the diagram) from the infected cell to the extraas depicted in the diagra
cellular environment $(B)$.

It is proposed that membrane fusion occurs at ites where the parasitophorous vacuolar membrane and the erythrocyte plasma membrane are in close proximity. Such places could be regions where vesicles and large tubules derived from the parasitophorous vacuolar membrane extend to the erythrocyte membrane in the form of a membranous network (Helmendorf \& Haldar 1993, Elford \& Ferguson 1993). Since protease inhibitors are (nown to intere with the release of parasites (Banyal 1986), the f Hoy et al. 1983, Lyon \& by by cleavage of selected red cell monbrane-skeleton compons out of the host cell by rant merezorion.

This model of merozoite release in $P$. Thiparu infected ery hrocytes wold im falciparming ite release and the formation of a duct-like structure could be highly regulated and coordinated to specific events of the parasite cell cycle. Studying he molecular components of this machinery could define new strategies for controlling infections in individuals afflicted with malaria disease. These findings might provide a conceptual framework to investigate whether evolutionary related organisms also have evolved similar mechanisms for parasite escape.

ACKNOWLEDGMENTS

To Dr Moises Wasserman for allowing us to carry out the initial observations in his laboratory. To Dr Danie Branton, Dr Vladimir Corredor, and Dr

\section{REFERENCES}

Andrews NW 1994. From lysosomes into the cytosol: the intracellular pathway of Trypanosoma cru Brazilian J Med Biol Res 27: 471-475.

Atkinson CT, Aikawa M 1990. Ultrastructure of malaria-infected erythrocytes. Blood Cells 16: 351-368 Banyal HS, Misra GC, Gupta CM, Dutta GP 1981. Involvement of malaria proteases in the interPlasmodium knowlesi infections. $J$ Parasite 67 : 623-626.

Deguercy A, Hommel M, Schrével J 1990. Purification and characterization of 37-kilodalton proteases from Plasmodium falciparum and Plasmodium berghei which cleave erythrocyte cytoskeletal components. Mol Biochem Parasito 38. 233-244.

1995. Origins of the parasitophorous vacuole membrane of the malaria parasite: surface area of the parasited red cell. Eur J Cell Biol 68: 446-449.

Dvorak JA, Miller LH, Whitehouse WC, Shiroishi T 1975. Invasion of eryth
Science 187: 748-749.

Elford BC, Ferguson DJP 1993. Secretory processes in
E Plasmodium. Parasitol Today 9: 80-81.

1985. to small hydrophobic solutes of humen erythrobility infected with Plasmodium falciparum. Mol Biochem Parasitol 16: 43-60.

Gruenberg J, Allred D, Sherman IW 1983. Scanning electron microscope-analysis of the protrusions (knobs) present on the surface of Plasmodium falciparumprese sion of rhesus erythrocytes by 983 . Studies on invaence of protease inhibitors. Exp Parasitol 55: 306ence

Imendorf HG, Haldar K 1993. Secretory transport in Plasmodium. Parasitol Today 9: 98-102.

Hermentin P, Enders B 1984. Erythrocyte invasion by malaria (Plasmodium falciparum) merozoites: recent Inst Mitt 76: 121-141.

ibbs AR, Stenzel DJ, Saul A 1997. Macromolecular Biol 72: 182-188. 
Jensen JB 1978. Concentration from continuous culture of erythrocytes infected with trophozoites and schizonts of Plasmodium falciparum. Am J Trop Hyg 27: 1274-1276.

Kara UAK, Stenzel DJ, Ingram LT, Kidson C 1988 The parasitophorous vacuole membrane of Plasmodium falciparam: demonstration of vesicle format7.

ambros C, Vanderberg JP 1979. Synchronization of Plasmodium falciparum erythrocytic stages in cul-

ture. J Parasitol 65: 418-420.
Langreth SG, Jensen JB, Reese RT, Trager W 1978. Fine structure of human malaria in vitro. J Parasito 25: $443-452$

Lyon JA, Haynes JD 1986. Plasmodium falciparum antigens synthesized by schizonts and stablilized presence of protease inhibitors. I Immunol 136 : 2245-2251.

Miller LH, Good MF, Milton G 1994. Malaria patho-

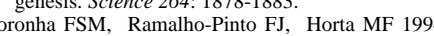
Identification of a putative pore-forming hemolysi active at acid $\mathrm{pH}$ in Leishmania amazonensis. Brasilian J Med Biol Res 27: 477-482. RL, Thomas AP, Taraschi TF 1991. Direct access to serum macromolecules by intraerythrocytic malaria parasite. Nature 353: 73-75.

Impson GL, Born JL, Cain G 1981. Growth of human is ager W, Jensen JB 1976. Human

W. Jensen JB 1976. Human malaria parasites in Ward GE, Miller LH, Dvorak JA 1993. The origin of parasitophorous vacuole membrane lipids in malariainfected erythrocytes. J Cell Sci 106: 237-248.

Winograd E, Sherman IW 1989. Characterization of a modified red cell membrane protein expressed on site Plasmodium falciparum: pocsible rale as a cytoadherent mediating protein. $J$ Cell Biol $108: 23$ 30.

Yamada KA, Sherman IW 1980. Plasmodium lophurae: malaria induced nucleotide changes in duckling (Anas domesticus) erythrocytes. Mol Biochem Parasitol 1: 187-198. 